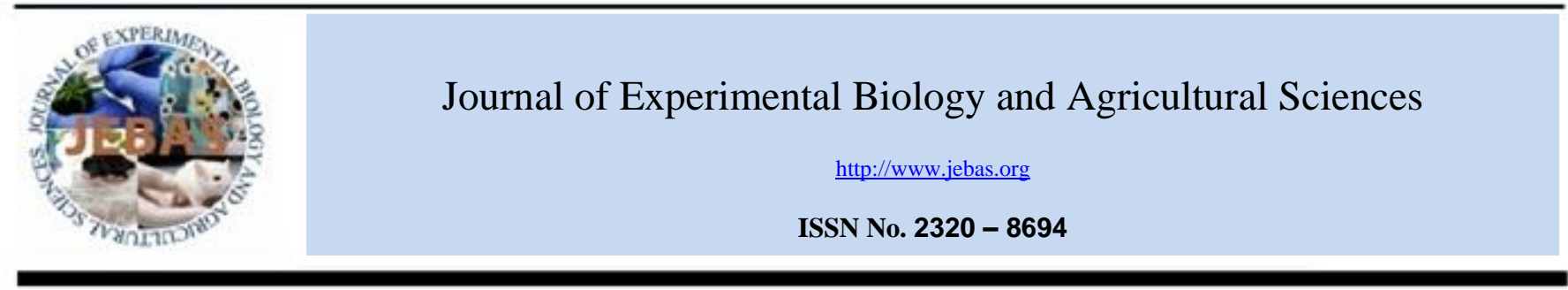

\title{
RESISTOTYPES OF Rhodococcus equi ISOLATED FROM FOALS WITH RESPIRATORY PROBLEMS
}

\author{
Sourabh Chhabra ${ }^{1}$, Khurana $\mathrm{S} \mathrm{K}^{2, *}$, Kapoor P K ${ }^{1}$ and Richa Khirbat ${ }^{3}$ \\ ${ }^{1}$ Department of Veterinary Public Health and Epidemiology, LUVAS, Hisar, Haryana, India 125004 \\ ${ }^{2}$ National Research Centre on Equines, Hisar, Haryana, India 125001 \\ ${ }^{3}$ Department of Animal Biotechnology, LUVAS, Hisar, Haryana, India 125004
}

Received - April 18, 2016; Revision - April 25, 2016; Accepted - May 21, 2016

Available Online - May 25, 2016

DOI: http://dx.doi.org/10.18006/2016.4(3S).242.248

\begin{abstract}
KEYWORDS
Rhodococcus equi

Foal

Resistotype

Marker

ABSTRACT

Rhodococcus equi has been recognized primarily as a respiratory pathogen of equines particularly of foals between one and four months of age. $R$. equi is ranked among the most important disease problems in equines especially because of its high prevalence and mortality rate. $R$. equi being an intracellular pathogen is very fastidious and requires prolonged specific antibiotic combination therapy lasting up to three months for successful treatment. This assumes further importance as no effective vaccination is available for prevention. It has zoonotic potential and may be responsible for infection in immunocompromised humans. This study is aimed at analyzing the distribution pattern of the resistotypes (R-types) of various isolates of $R$. equi in different areas of Haryana and Rajasthan, India. Antimicrobial susceptibility pattern of $R$. equi isolates was determined by Kirby Bauer disc diffusion method following the Clinical and Laboratory Standards Institute (CLSI) guidelines. A total of twenty eight clinical isolates of $R$. equi from foals from different parts of Haryana and Rajasthan were used in this study. Antibiogram of $R$. equi isolates with 33 antibiotics revealed ten distinct R-types: R-type 1 to R-type 10, on the basis of variable results of four antibiotics i.e. amoxycillin, gentamycin, colistin and streptomycin. Out of ten resistotypes obtained the relative frequencies of R-1 resistotype and R-4 resistotype were found to be high i.e. $28.57 \%$ and $25 \%$, respectively. Differentiation of $R$. equi strains into R-types is an important tool for therapeutics. In addition to direct foal management, it may have implication in identifying the source and spread of infection, and as epidemiological marker to correlate various isolates from various places, ultimately helping in therapeutics for timely control.
\end{abstract}

* Corresponding author

E-mail: sandipkk2003@yahoo.co.in (Khurana S K)

Peer review under responsibility of Journal of Experimental Biology and Agricultural Sciences.

Production and Hosting by Horizon Publisher India [HPI] (http://www.horizonpublisherindia.in/).

All rights reserved.
All the article published by Journal of Experimental Biology and Agricultural Sciences is licensed under a Creative Commons Attribution-NonCommercial 4.0 International License Based on a work at www.jebas.org. 


\section{Introduction}

Rhodococcus equi is aerobic, non-sporulating, non-motile, gram-positive bacteria, found in soil, herbivore dung and the intestinal tract of cattle, horse, sheep, pig and some other animals. It is primarily a respiratory pathogen mainly affecting the foals between 1-4 months of age (Khurana et al., 2015). The disease is direct anthropozoonosis since the animals are primary reservoirs of the etiological agent (Khurana, 2014). It can be responsible for infection in humans compromised by immunosuppressive drug therapy, lymphoma or AIDS (Takai et al., 1995; Mizuno et al., 2005; Napoleao et al., 2005). $R$. equi has been ranked one of the most important disease problems in the horse industry especially because of its high prevalence and mortality rate (Blood-Horse, 2009; Muscatello, 2009; Elissalde et al., 1980; Mir et al., 2015).

The symptoms in foals include pyrexia, respiratory distress, pulmonary lesions and chronic lung abscesses, which when left untreated lead to death due to asphyxiation (Wichtel et al., 1991; Lavoie et al., 1994). The infection can spread from the lungs to other organs and joints when granulomatous foci in the lung open up (Prescott, 1991; Giguere, et al., 2011). It has been reported that only a small proportion of all $R$. equi in soil are able to cause the infection and only $R$. equi carrying virulence plasmids can cause disease in foals (Muscatello et al., 2006). Foal survival requires successful antimicrobial therapy (Sweeny et al., 1987).

Most of the conventional antibiotics are not effective against $R$. equi because it is an intracellular pathogen. $R$. equi is usually resistant to beta-lactam antibiotics such as penicillin G, oxacillin, ampicillin, carbenicillin and cefazolin (Kedlaya et al., 2001). Current treatment comprises the use of a combination of erythromycin and rifampin (Jacks, 2003).
This combination facilitates the drugs to penetrate the lung abscesses, macrophages or neutrophils where the bacteria survive and multiply (Prescott, 1991). During the last decade the minimum inhibitory concentrations (MIC) of erythromycin and rifampin for $R$. equi are rising and there are reports of resistance to these antibiotics (von Bargen \& Haas, 2009). Therapy with orally administered macrolides has greatly improved survival rates for foals with $R$. equi pneumonia (Sweeney et al., 1987). $R$. equi isolates resistant to the commonly used macrolides (azithromycin, clarithrimycin and erythromycin) as well as rifampin are emerging these days. The overall prevalence of $R$. equi isolates resistant to macrolides or rifampin has been reported as $4 \%$ (Giguere et al., 2010). Keeping in view, the emerging variable resistance to various important antibiotics and difficulty in identification of $R$. equi isolates due to inconsistent biochemical tests, the present study was undertaken to study the resistance pattern of various isolates of R. equi and to find out various resistotypes.

\section{Materials and Methods}

A total of twenty eight $R$. equi isolates from foals with respiratory problems from various parts of Haryana and Rajasthan were used. Nasal swabs from each case were collected in Cary-Blair transport medium and processed in the laboratory for isolation. All the isolates of $R$. equi were maintained on nutrient agar slants. All the twenty eight isolates of $R$. equi were identified by cultural characteristics, gram staining and biochemical tests. After $48 \mathrm{hrs}$ incubation of nutrient agar plates characteristic 1-2 mm irregularly round smooth, mucoid, glistening, semi transparent, salmon pink to yellow coloured and coalescing colonies on nutrient agar were observed. The antibiotic sensitivity test was conducted by Kirby Bauer disc diffusion method following the Clinical and Laboratory Standards Institute (CLSI) guidelines (Bauer et al., 1966).

Table 1 Antibiotics used and their concentration.

\begin{tabular}{|llcccc|}
\hline S. No & Antibiotics & Concentration & S. No & Antibiotics & Concentration \\
\hline 1 & Azithromvcin & $30 \mathrm{mcg}$ & 18 & Ampicillin & $25 \mathrm{mcg}$ \\
\hline 2 & Ciprofloxacin & $10 \mathrm{mcg}$ & 19 & Cloxacillin & $30 \mathrm{mcg}$ \\
\hline 3 & Chloramphenicol & $25 \mathrm{mcg}$ & 20 & Kanamvcin & $05 \mathrm{mcg}$ \\
\hline 4 & Ceftriaxone & $30 \mathrm{mcg}$ & 21 & Nalidixic acid & $30 \mathrm{mcg}$ \\
\hline 5 & Cefaparazone & $75 \mathrm{mcg}$ & 22 & Oxacillin & $05 \mathrm{mcg}$ \\
\hline 6 & Enrofloxacin & $10 \mathrm{mcg}$ & 23 & Penicillin G & $10 \mathrm{mcg}$ \\
\hline 7 & Ervthromycin & $10 \mathrm{mcg}$ & 24 & Sulphadiazine & $100 \mathrm{mcg}$ \\
\hline 8 & Methicillin & $10 \mathrm{mcg}$ & 25 & Trimethoprim & $25 \mathrm{mcg}$ \\
\hline 9 & Norfloxacin & $10 \mathrm{mcg}$ & 26 & Amoxvcillin & $10 \mathrm{mcg}$ \\
\hline 10 & Neomvcin & $03 \mathrm{mcg}$ & 27 & Colistin & $25 \mathrm{mcg}$ \\
\hline 11 & Ofloxacin & $02 \mathrm{mcg}$ & 28 & Gentamvcin & $30 \mathrm{mcg}$ \\
\hline 12 & Oxvtetracycline & $30 \mathrm{mcg}$ & 29 & Streptomvcin & $25 \mathrm{mcg}$ \\
\hline 13 & Rifampicin & $30 \mathrm{mcg}$ & 30 & Tetracvcline & $10 \mathrm{mcg}$ \\
\hline 14 & Roxithromvcin & $30 \mathrm{mcg}$ & 31 & Lincomvcin & $15 \mathrm{mcg}$ \\
\hline 15 & Tobramvcin & $30 \mathrm{mcg}$ & 32 & Pefloxacin & $05 \mathrm{mcg}$ \\
\hline 16 & Vancomycin & $05 \mathrm{mcg}$ & 33 & Clindamvcin & $25 \mathrm{mcg}$ \\
\hline 17 & Amikacin & $30 \mathrm{mcg}$ & & & \\
\hline
\end{tabular}

mcg = Micrograms 
A few colonies of the $R$. equi were picked with a wire loop from the nutrient agar slant and inoculated into a test tube containing brain heart infusion broth (BHI). These tubes were incubated at $37^{\circ} \mathrm{C}$ for $16-18$ hours to produce a bacterial suspension of moderate cloudiness. The bacterial broth suspension was spread evenly onto the surface of Muellar Hinton Agar (MHA) plates covering whole of the plate with a sterile cotton swab. After the inoculums had dried, the disks were placed on the agar. The discs of thirty three antibiotics were used (Table 1).

Plates were then incubated immediately at $37^{\circ} \mathrm{C}$ for $24 \mathrm{hrs}$. After incubation, the diameter of zone of inhibition was measured with antibiotic zone reader and interpreted as per manufacturer interpretation values. The results of antibiotic sensitivity were analyzed to find out antibiotics which are sensitive or resistant to all isolates. Antibiotics which were showing variable results i.e. some of isolates were sensitive and some were resistant to antibiotics were identified. On the basis of antibiotic sensitivity/ resistance twenty eight $R$. equi isolates were classified into various resistotypes.

\section{Results}

All the isolates were sensitive to 20 antibiotics (zone range with mean in $\mathrm{mm}$ ): azithromycin (30-34, 32), ciprofloxacin(31-38, 34.5), chloramphenicol (20-30, 25), ceftriaxone $(28-34,31)$, clindamycin $(20-28,24)$, cefaparazone
(22-30, 26), enrofloxacin $(28-34,31)$, erythromycin (32-38, $35)$, lincomycin $(30-34,32)$, methicilin $(20-28,24)$, norfloxacin $(21-30,25.5)$, neomycin $(20-32,26)$, ofloxacin (24-32, 28), oxytetracycline $(10-19,14.5)$, pefloxacin $(25-31$, 28), rifampicin (31-36, 33.5), roxithromycin(31-37, 34), tetracycline $(25-33,29)$, tobramycin $(20-34,27)$, vancomycin $(22-28,24)$. All the isolates were resistant showing no inhibition zone or zone of inhibition within resistant range to 9 antibiotics viz. amikacin, ampicillin, cloxacillin, kanamycin, nalidixic acid, oxacillin, penicillin-G, sulphadiazine, trimethoprim. However, 4 antibiotics viz., amoxycillin, colistin, gentamycin, streptomycin showed variable results (Table 2) which formed the basis to formulate resistotypes of R. equi. Ten resistotypes could be detected amongst 28 isolates of $R$. equi (Table 3). The resistotype R-1 represented eight isolates (NS-1, NS-44, NS-98, NS-117, NS-120, NS-151, NS244, NS-290) which showed $28.50 \%$ relative frequency and found to be resistant to amoxycillin, gentamycin, streptomycin and sensitive to colistin. Resistotype R-2 represented only one isolate (NS-6) which showed $03.57 \%$ relative frequency and found to be sensitive with all the four antibiotics, Resistotype R-3 represented two isolates (NS-25, NS-276) which showed $07.14 \%$ relative frequency and found to be resistant to all four anitibiotics, Resistotype R-4 represented seven isolates (NS36, NS-79, NS-113, NS-150, NS-231, FNS-4, FNS-5) which showed $25 \%$ relative frequency and found to be resistant to gentamycin, streptomycin and sensitive to amoxycillin, colistin.

Table 2 Antibiotic sensitivity of twenty eight isolates of Rhodococcus equi.

\begin{tabular}{|c|c|c|}
\hline \multicolumn{2}{|c|}{ Antibiotics to which all the isolates were } & \multirow{2}{*}{$\begin{array}{l}\text { Antibiotics to which isolates showed variable resistance } \\
\text { pattern }\end{array}$} \\
\hline Sensitive & Resistant & \\
\hline Azithromycin & Amikacin & Amoxycillin (12S, 16 R) \\
\hline Ciprofloxacin & Ampicillin & Colistin (21S, 7R) \\
\hline Chloramphenicol & Cloxacillin & Gentamycin (4S, 24R) \\
\hline Ceftriaxone & Kanamycin & Streptomycin (5S, $23 \mathrm{R})$ \\
\hline Clindamycin & Nalidixic acid & \\
\hline Cefaparazone & Oxacillin & \\
\hline Enrofloxacin & Penicillin-G & \\
\hline Erythromycin & Sulphadiazine & \\
\hline Lincomycin & Trimethoprim & \\
\hline \multicolumn{3}{|l|}{ Methicilin } \\
\hline \multicolumn{3}{|l|}{ Norfloxacin } \\
\hline \multicolumn{3}{|l|}{ Neomycin } \\
\hline \multicolumn{3}{|l|}{ Ofloxacin } \\
\hline \multicolumn{3}{|l|}{ Oxytetracycline } \\
\hline \multicolumn{3}{|l|}{ Pefloxacin } \\
\hline \multicolumn{3}{|l|}{ Rifampicin } \\
\hline \multicolumn{3}{|l|}{ Roxithromycin } \\
\hline \multicolumn{3}{|l|}{ Tobramycin } \\
\hline \multicolumn{3}{|l|}{ Tetracycline } \\
\hline Vancomycin & & \\
\hline
\end{tabular}

*Number in parentheses represent sensitive (S) and resistant (R) isolates 
Table 3 Resisotypes recognized amongst twenty eight Rhodococcus equi isolates.

\begin{tabular}{|c|c|c|c|c|c|c|}
\hline \multirow[t]{2}{*}{ Resitotype } & \multicolumn{4}{|c|}{ Sensitive or Resistant to antibiotic } & \multirow[t]{2}{*}{ Sample No. (No. of Isolates) } & \multirow[t]{2}{*}{ Relative frequency } \\
\hline & $\mathrm{A}$ & $\mathrm{C}$ & $\mathrm{G}$ & Str & & \\
\hline $\mathrm{R}-1$ & $\mathrm{R}$ & $\mathrm{S}$ & $\mathrm{R}$ & $\mathrm{R}$ & $\begin{array}{l}\text { NS-1, NS-44, NS-98, NS-117, NS-120, NS-151, } \\
\text { NS-244 NS-290 (8) }\end{array}$ & $28.57 \%$ \\
\hline $\mathrm{R}-2$ & $\mathrm{~S}$ & $\mathrm{~S}$ & $\mathrm{~S}$ & $\mathrm{~S}$ & NS-6 (1) & $03.57 \%$ \\
\hline $\mathrm{R}-3$ & $\mathrm{R}$ & $\mathrm{R}$ & $\mathrm{R}$ & $\mathrm{R}$ & NS-25, NS-276 (2) & $07.14 \%$ \\
\hline $\mathrm{R}-4$ & $\mathrm{~S}$ & $\mathrm{~S}$ & $\mathrm{R}$ & $\mathrm{R}$ & $\begin{array}{l}\text { NS-36, NS-79, NS-113, NS-150, NS-231, FNS- } \\
4, \text { FNS-5 (7) }\end{array}$ & $25.00 \%$ \\
\hline R-5 & $\mathrm{R}$ & $\mathrm{S}$ & $\mathrm{S}$ & $\mathrm{R}$ & NS-48, NS-216 (2) & $07.14 \%$ \\
\hline R-6 & $\mathrm{S}$ & $\mathrm{S}$ & $\mathrm{R}$ & $\mathrm{S}$ & NS-62 (1) & $03.57 \%$ \\
\hline $\mathrm{R}-7$ & $\mathrm{R}$ & $\mathrm{R}$ & $\mathrm{R}$ & $\mathrm{S}$ & NS-77 (1) & $03.57 \%$ \\
\hline $\mathrm{R}-8$ & $\mathrm{~S}$ & $\mathrm{R}$ & $\mathrm{R}$ & $\mathrm{R}$ & NS-121, NS-161, NS-288 (3) & $10.71 \%$ \\
\hline R-9 & $\mathrm{R}$ & $\mathrm{R}$ & $\mathrm{S}$ & $\mathrm{R}$ & NS-170 (1) & $03.57 \%$ \\
\hline $\mathrm{R}-10$ & $\mathrm{R}$ & $\mathrm{S}$ & $\mathrm{R}$ & $\mathrm{S}$ & NS-202, NS-188 (2) & $07.14 \%$ \\
\hline
\end{tabular}

$\mathrm{A}=$ Amoxycillin, $\mathrm{C}=$ Colistin, $\mathrm{Str}=$ Streptomycin, $\mathrm{G}=$ Gentamycin, $\mathrm{R}=$ Resistant, $\mathrm{S}=$ Sensitive

Resistotype R-5 represented two isolates (NS-48, NS-216) which showed $03.57 \%$ relative frequency and found to be resistant to amoxycillin, streptomycin and sensitive to gentamycin, colistin., Resistotype R-6 represented one isolate NS-62) which showed $03.57 \%$ relative frequency and found to be resistant to gentamycin and sensitive to amoxycillin, colistin, streptomycin.

Resistotype R-7 represented one isolate (NS-77) which showed $03.57 \%$ relative frequency and found to be resistant to amoxycillin, gentamycin, colistin and sensitive to streptomycin.

Resistotype R-8 represented three isolates (NS-121, NS-161, NS-288) which showed $10.71 \%$ relative frequency and found to be resistant to gentamycin, streptomycin, colistin and sensitive to amoxycillin. Resistotype R-9 represented one isolate (NS-170) which showed $03.57 \%$ relative frequency and found to be resistant to amoxycillin, colistin, streptomycin and sensitive to gentamycin, Resistotype R-10 represented two isolates (NS-202, NS-188) which showed $07.14 \%$ relative frequency and found to be resistant to amoxycillin, gentamycin and colistin, streptomycin ( Figure 1). Out of ten resistotypes obtained the relative frequencies of R-1 resistotype and R-4 resistotype were higher i.e. $28.57 \%$ and $25 \%$, respectively. Resistotype R-1 included eight isolates of $R$. equi which were isolated from nasal swabs of foals belonging to various places i.e. Tohana (NS-1), Sorkhi (NS-44), Gangua (NS-98), Hanumangarh (Rajasthan) (NS-177), Ismaila (NS120), Muklan (NS-151), Barwala (NS-244) and Kaimri (NS290). Whereas resistotypes R-4 included isolates of $R$. equi from various places viz. Sorkhi (NS-36), Sachakhera (NS-79), Muklan (NS-113 and 150), Jakhal (NS-231) and Tohana (FNS4 and FNS 5). Four isolates of $R$. equi of Tohana were characterized into three resistotypes viz. R-1 (NS-1), R-2 (NS6) and R-4 (FNS-4 and FNS-5).

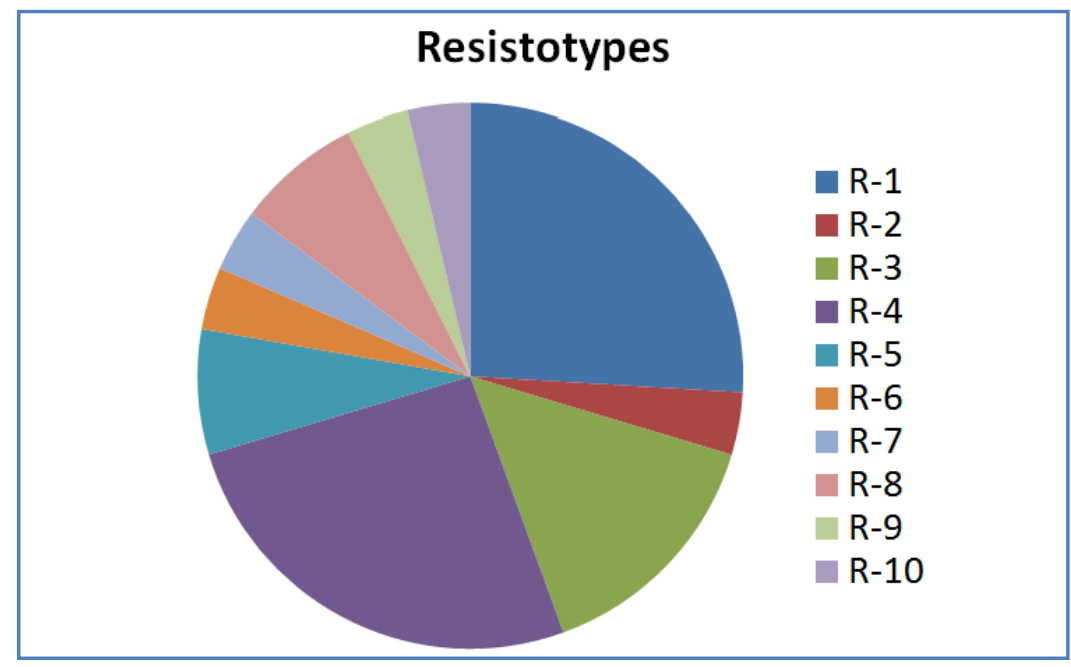

Figure 1 Proportion of resistotypes of $R$. equi isolates. 


\section{Discussion and conclusions}

For selection of appropriate agents to treat $R$. equi infections, both the in vivo and in vitro properties of the antimicrobial agents should be considered. Previous reports have shown that $R$. equi is susceptible to ampicillin/sulbactam, amoxycillin/clavulanic acid, gentamycin, erythromycin, tetracycline, rifampin, TMP-SMZ, imipenem, and vancomycin . The organism is usually less susceptible to penicillin, ampicillin, cephalosporins, or quinolones (Prescott, 1991; McNeil \& Brown 1992; Nordmann \& Ronco 1992; Giguere et al., 2011).

All the twenty eight isolates of $R$. equi of this study were grouped into ten resistotypes on the basis of four antibiotics which showed variable results described vide supra. A total of sixteen resistotypes $\left(2^{n}=2^{4}=2 \times 2 \times 2 \times 2=16\right)$ could be possible on the basis of four variable antibiotics if larger number of isolates could be studied for resistotyping. These results could not be compared to previous reports because of non availability of such type of study in earlier literature which shows that the present study is probably the first study of resistotypes of $R$. equi. However, in recent review Khurana (2015) reported emergence of antibiotic resistance to various antibiotics. Resistance to rifampicin in $R$. equi attributatable to monooxygenase like sequence has been reported (Anderson et al., 1997). Mutations in rpoB gene leading to rifampicin resistance have been reported (Asoh et al., 2013; Liu et al., 2014).

These resistotypes could be used as epidemiological marker during outbreak studies due to $R$. equi in equines. In this study Resistotype R-1 represented eight isolates (NS-1, NS-44, NS98, NS-117, NS-120, NS-151, NS-244, NS-290) which showed highest relative frequency $(28.50 \%)$ and found to be resistant to amoxycillin, gentamycin, streptomycin and sensitive to colistin. These isolates were from various places i.e. Tohana (NS-1), Sorkhi (NS-44), Gangwa (NS-98), Hanumangarh, Rajasthan (NS-177), Ismaila (NS-120), Muklan (NS-151), Barwala (NS-244) and Kaimri (NS-290).

All these isolates were from the areas near Hisar city within a radius of $70 \mathrm{~km}$ except isolate NS-177 from Hanumangarh, Rajasthan. Resistotype R-4 had second highest relative frequency (25\%) represented by seven isolates (NS-36, NS-79, NS-113, NS-150, NS-231, FNS-4, FNS-5) resistant to gentamycin, streptomycin and sensitive to amoxycillin, colistin from various places viz. Sorkhi (NS-36), Sacchakhera (NS-79), Muklan (NS-113 and 150), Jakhal (NS-231) and Tohana (FNS4 and FNS 5). Four isolates of $R$. equi of Tohana were characterized into three resistotypes viz. R-1 (NS-1), R-2 (NS6) and R-4 (FNS-4 and FNS-5). This finding is very interesting and showing the use of resistotypes as epidemiological marker and could be useful for therapy in a particular endemic area/ farm after identifying the resistotype.

In the present study all isolates of $R$. equi were found sensitive to rifampicin and macrolides antibiotics viz. azithromycin, erythromycin and roxithromycin. Macrolide resistance in $R$. equi has also been reported (Burton et al., 2013; Liu et al., 2014). A glycopeptides resistance operon vanO having potential implications in $R$. equi therapy has been described (Gudeta et al., 2014). Cohen (2014) has warned about the challenges of emergence of resistance to macrolide due to nonavailability of effective alternative for $R$. equi therapeutics.

Rifampicin along with macrolide is drug of choice for effective treatment of $R$. equi infections. However the emergence of resistance against these antibiotics poses a serious challenge in therapeutic management. But in this study such problem has not been observed, therefore the cases of foals respiratory diseases can be treated successfully in this area with standard antibiotic combination therapy used to treat R.equi infections. It is therefore, recommended that veterinarians must use antibiotics for treatment judiciously.

\section{Acknowledgements}

The authors are thankful to National Research Centre on Equines, Hisar and Lala Lajpat Rai University of Veterinary and Animal Sciences, Hisar for providing necessary facilities to carry out the research work.

\section{Conflict of interest}

Authors would hereby like to declare that there is no conflict of interests that could possibly arise.

\section{References}

Anderson SJ, Quan S, Gowan B, Dabbs ER (1997) Monooxygenase like sequence of Rhodococcus equi gene conferring increased resistance to rifampin by inactivating this antibiotic. Antimicrobial Agents and Chemotherapy $41: 218$ 221.

Asoh N, Watanabe H, Fines-Guyon M, Watanabe K., Oishi K, Kositsakulchai W, Sanchai T, Kunsuikmengrai K, Kahintapong S, Khanawa B, Tharavichitkul P, Sirisanthana T, Nagatake $T$ (2013) Emergence of rifampin-resistant Rhodococcus equi with several types of mutations in rpo B gene among AIDS patients in northern Thailand. Journal of Clinical Microbiology 41: 2337-2340. doi: 10.1128/JCM.41.6.2337-2340.2003.

Bauer AW, Kirby WMM, Sherris JC, Turck M (1966) Antibiotic susceptibility testing by a standardized single disk method. American Journal of Clinical Pathoogy 45:493-6.

Blood-Horse (2009) Rhodococcus.

http://cs.bloodhorse.com/blogs/blood-horsepressroom/archive/2009/03/05/.

Burton AJ, Giguère S, Sturgill TL, Berghaus LJ, Slovis NM, Whitman JL, Levering C, Kuskie KR, Cohen ND (2013) Macrolide-and Rifampin- Resistant Rhodococcus equi on a 
Horse Breeding Farm, Kentucky, USA. Emerging Infectious Diseases 19 : 282-285. doi: 10.3201/eid1902.121210.

Cohen ND (2014) Rhodococcus equi foal pneumonia. Veterinary Clinics: Equine Practice 30: 609-622. doi: http://dx.doi.org/10.1016/j.cveq.2014.08.010.

Elissalde GS, Renshaw HW Walserg JA (1980) Corynebacterium equi. Comparative Immunology Microbiology and Infectious Diseases 3: 433-445.

Giguere S, Lee E, Cohen ND (2010) Prevalence of Rhodococcus equi isolate resistant to macrolides or rifampicin and outcome of infected foals . Journal of American Veterinary Medical Association 237: 74-81. doi: 10.2460/javma.237.1.74.

Giguère S, Cohen ND, Keith Chaffin M, Slovis NM, Hondalus MK, Hines SA, Prescott JF (2011) Diagnosis, treatment, control, and prevention of infections caused by Rhodococcus equi in foals. Journal of Veterinary Internal Medicine 25 : 1209-1220. doi: 10.1111/j.1939-1676.2011.00835.x.

Gudeta DD, Moodley A, Borotolaia V, Guardabassi L (2014) $\operatorname{van} \mathrm{O}$, a new glycopeptides resistance operon in environmental Rhodococcus equi isolates. Antimicrobiai Agents and Chemotherapy 58 : 1768-1770. doi: 10.1128/AAC.01880-13.

Jacks S (2003) In vitro susceptiblities of Rhodococcus and other common equine pathogens to azithromycin, clarithromycin, and 20 other antimicrobials. Antimicrobial Agents and Chemotherapy 47: 1742-1745. doi: 10.1128/AAC.47.5.1742-1745.2003.

Kedlaya I, Ing MB, Wong SS (2001) Rhodococcus infections in immunocompetent hosts: case report and review. Clinical Infectious Diseases 32 : 39-46. doi: 10.1086/318520.

Khurana SK (2014) Rhodococcus equi infection. In: SR Garg (Ed.) Zoonoses: bacterial Diseases. Daya Publishing House. New Delhi. India. Pp. 390-401.

Khurana SK (2015) Current understanding of Rhodococcus equi infection and its zoonotic implications. Advances in Animal and Veterinary Sciences 3 :1-10. http://dx.doi.org/10.14737/journal.aavs/2015/3.1.1.10.

Khurana SK, Singha H, Malik P, Gulati BR, Singh RK (2015) Molecular characterization of clinical isolates of Rhodococcus equi with PCR assay based on virulence plasmid marker. Indian Journal of Animal Science 85 : 1063-1066.

Lavoie JP, Fiset L, Laverty S (1994) Review of 40 cases of lung abscesses in foals and Adult Horses. Equine Veterinary Journal $26 \quad$ : $\quad 348-352$ DOI: $10.1111 /$ j.20423306.1994.tb04401.x.
Liu H, Wang Y, Yan J, Wang C, He H (2014) Appearance of multidrug-resistant virulent Rhodococcus equi clinical isolates obtained in China. Journal of Clinical Microbiology 52 : 703. doi: 10.1128/JCM.02925-13.

McNeil MM, Brown JM (1992) Distribution and antimicrobial susceptibility of Rhodococcus equi from clinical specimens. European Journal of Epidemiology 8: 437-443.

Mir IA, Kumar B, Taku A, Bhardwaj RK, Bhat MA, Badroo GA (2015) Prevalence and Antibiogram study of Rhodococcus equi in equines of Jammu and Kashmir, India. Journal of Equine Science 26 : 21-24. doi: 10.1294/jes.26.21.

Mizuno Y, Sato F, Sakamoto M, Yoshikawa K, Yoshida M, Shiba K, Onodera S, Matsuura R, Takai SJ (2005) VapBpositive Rhodococcus equi infection in an HIV-infected patient in Japan. Journal of Infection and Chemotherapy $11: 37-40$. doi: http://dx.doi.org/10.1007/s10156-004-0355-X.

Muscatello G, Anderson GA, Gilkerson JR, Browning GF (2006) Associations between the ecology of virulent Rhodococcus and the epidemiology of R-equi pneumonia on Australian thoroughbred farms. Applied and Environmental Microbiology 72 : 6152-6160. doi: 10.1128/AEM.00495-06.

Muscatello G (2009) Detection of Virulent Rhodococcus in Exhaled Air Samples from Naturally Infected Foals. Journal of Clinical Microbiology 47: 734-737. doi:10.1128/JCM.0139508 .

Napolea o F, Damasco PV, Camello TCF, do Vale MD, de Andrade AFB, Hirata Jr. R, de Mattos-Guaraldi AL (2005) Pyogenic liver abscess due to Rhodococcus equi in an immunocompetent host. Journal of Clinical Microbiology 43:1002-1004. doi: 10.1128/JCM.43.2.1002-1004.2005.

Nordmann P, Ronco E (1992) In vitro antimicrobial susceptibility of Rhodococcus equi. The Journal of Antimicrobial Chemotherapy 29: 383-93. doi: 10.1093/jac/29.4.383.

Prescott JF (1991) Rhodococcus equi - An Animal and Human Pathogen. Clinical Microbiology Reviews 4 : 20-34. doi: 10.1128/CMR.4.1.20.

Sweeny CR, Sweeny RW, Divers TJ (1987) Rhodococus equi pneumonia in 48 foals: response to antimicrobial therapy . Veterinary Microbiology 14: 329-336. doi:10.1016/03781135(87)90120-9.

Takai S, Imai Y, Fukunaga N, Uchida Y, Kamisawa K, Sasaki Y, Tsubaki S, Sekizaki T (1995) Identification of virulenceassociated antigens and plasmids in Rhodococcus equi from patients with AIDS. Journal of Infectious Diseases 172: 13061311. doi: 10.1093/infdis/172.5.1306 
von Bargen K, Haas A (2009) Molecular and infection biology of the horse pathogen Rhodococcus. FEMS Microbiology Reviews $\quad 33 \quad$ : $\quad 870-891$ doi: $10.1111 /$ j.15746976.2009.00181.x.
Wichtel MG, Anderson KL, Johnson TV, Nathan U, Smith L (1991) Influence of age on neutrophil function in foals. Equine Veterinary Journal 23 : 466-469. doi: 10.1111/j.20423306.1991.tb03763.x. 